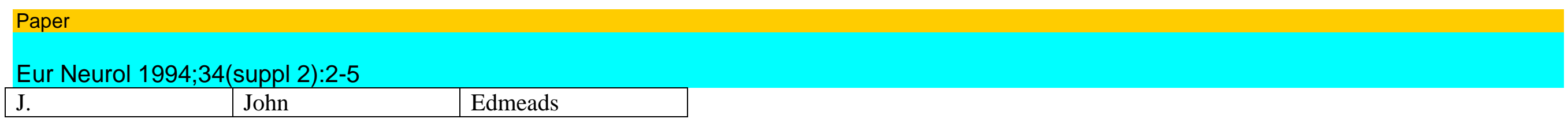

Department of Neurology, University of Toronto, Ont., Canada

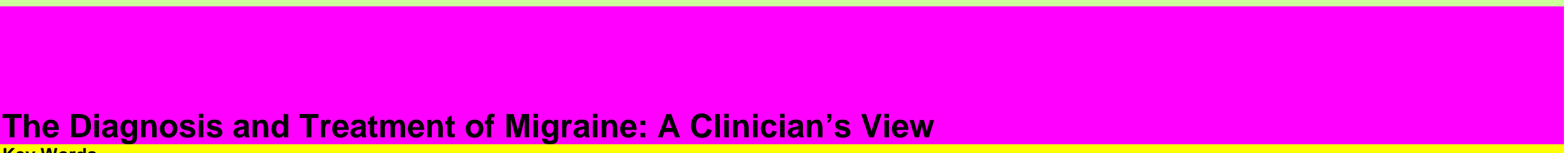

Key Words

Migraine

Diagnosis

Diagnosis

Treatment

Consultation

Abstract

The introduction of the International Headache Society (IHS) diagnostic criteria has made migraine a 'positive' diagnosis, not one of exclusion. Studies in Canada have shown that many sufferers do not consult their physician about their migraine, and that the use of over-the-counter and prescription analgesics is common. Treatment should comprise a physician-patient partnership based on a correct and accepted diagnosis, in which both participate in a programme of education about the condition, identification and reduction of migraine triggers, and appropriate use of non-pharmacological, prophylactic and acute pharmacological treatments. The pathogenesis of chronic daily headache is uncertain, but it is frequently associated with habitual overuse of simple analgesics and ergotamines.

John Edmeads, University of Toronto, Sunnybrook Health Science Centre, 2075 Bayview Avenue A460, North York, Ont. M4N 3M5 (Canada)

\title{
Introduction
}

When patients report headache, the clinician must determine the appropriate extent of investigation, make the correct diagnosis and prescribe the correct treatment. Although migraine is a common problem, it is often not recognized, and there are a number of issues concerning its diagnosis that deserve examination. The difficulties that beset migraine treatment involve not only the prescription of the most appropriate medications for those who present with migraine, but also the lack of efficacy of self-treatment by those sufferers who do not consult their doctors. Standard migraine therapies are used sub-optimally, and analgesic overdose and chronic daily headache are prevalent.

\section{Diagnosis of Migraine}

The introduction in 1988 of the International Headache Society (IHS) diagnostic criteria have made migraine a positive diagnosis, rather than a diagnosis of exclusion [1]. These detailed criteria are of particular relevance to those researching migraine and they can be summarized and simplified for clinical diagnosis (table 1). Careful questioning of the patient and application of these diagnostic criteria allows the clinician to distinguish between migraine and tension-type headache - an important distinction because the treatment for migraine is different from that for tension headache.

Implicit in the diagnostic criteria for migraine is the absence of historical or physical features of underlying disease. It is therefore necessary to obtain a detailed and consistent history and carry out a brief, but sensitive and highly focused, neurological examination (table 2). Examination of the head will rarely be of use in diagnosing the problem but can be very reassuring to patients.

Fulfilment of the IHS diagnostic criteria, along with a normal history and normal examination, should be sufficient to diagnose migraine. However, sometimes a patient presents with atypical migraine: for example, most of the attacks are 'normal', but occasionally a migraine attack is accompanied by symptoms such as numbness, tingling and weakness or speech disturbance. If there is anything

\section{(c) 1994 S. Karger AG, Basel}

$\$ 8.00 / 0$

unusual in the patient's history or neurological examination the clinician must then consider whether there is indeed some underlying ominous disease and decide what investigations are necessary. If the headache about which the patient is consulting is the first or worst experienced, there is particular need for a thorough investigation for the presence of a serious disorder. There are a number of 'danger signals' that should be looked for: accompanying fever may indicate an infection; neck or back pain could indicate pus or blood in the subarachnoid space; chronic malaise, myalgia and arthralgia (particularly in patients older than 50 years) suggest the presence of temporal arteritis; increasing drowsiness, cognitive dysfunction, clumsiness, weakness or lack of balance might be symptoms of increased intracranial pressure [2].

For patients, the headaches and symptoms associated with 'ordinary' migraine will frequently raise concerns about the presence of some underlying disease. The extent of the medical examination required is therefore an important issue in migraine diagnosis. Many patients in North America want the reassurance of a normal computerized axial tomography (CAT) scan. The clinician should aim to minimize the unnecessary use of further investigations, reserving CAT scans, magnetic resonance imaging and 
laboratory investigations for those headache patients with unusual signs and symptoms. The relationship between the clinician and patient is crucial here; it should be one in which the patient can feel confident in the clinician and the diagnosis.

\section{Chronic Daily Headache}

Difficulties in diagnosis can also arise in patients with chronic daily headache. In some patients who have migraine, as defined by the IHS criteria, the pattern of the headaches gradually changes over a period of months or years. The headaches become less episodic and instead the symptoms become continuous, with occasional peaks of migraine. The neurological examination shows nothing abnormal but the chronic daily headache will often be associated with dependence on simple analgesics or ergot-amines [3-6]. On waking with a headache, the patient takes acute medication. The headache is relieved, but as the medication wears off the headache returns and the patient takes more medication. Thus a dependence on or abuse of simple analgesics or ergotamines is set up. Withdrawal of the drug is frequently associated with severe rebound headaches [7], particularly with ergotamines $[3,5]$.

Table 1. Summary of IHS diagnostic criteria for migraine from Headache Classification Committee of the International Headache Society

$\begin{array}{ll}\text { Migraine } & \text { Lasts 4-72 } \mathrm{h} \\ \text { without aura } & \text { Has at least two of the following characteristics: }\end{array}$

unilateral pulsating

moderate or severe intensity aggravated by physical activity Associated with one or more of the following: nausea, vomiting, photophobia, phonophobia

Migraine $\quad$ Two or more headaches preceded by aur

with aura Aura symptoms usually involving:

blurred vision flashing lights missing area of visual field Aura symptoms fully reversible, lasting less than $1 \mathrm{~h}$. Headache follows aura within $1 \mathrm{~h}$

Reprinted with permission from [1].

Table 2. Summary of procedures for a differential diagnosis of migraine

Clinical history

Is this the first or worst headache the patient has experienced, or is

it the most recent of a series? If a series:

how long has the patient suffered from the attacks?

what is the timing and pattern of the attacks?

what is the duration of each attack? What is the nature of the headache? Are there accompanying symptoms? Are there any aggravating features? Does the patient use analgesics or ergotamines?

Examination

Assessment of cognitive state:

manner in which the patient gives the history

patient's behaviour Check heart, lungs, blood pressure and temperature With patient in a supine position, check nuchal rigidity Examine optic fundi for the presence of papilledema;

record pupillary reaction Check motor system:

facial movements symmetrical?

assess power in shoulders, hands, hips and feet

any drift of the outstretched arms with eyes closed? Test reflexes: biceps and knee jerks, plantar response Examine the head: normal contours, sore spots?

Reprinted with permission from Edmeads et all. [9].

Decrease in

central pain

Medication "wears off"

uppression of endogenous pain system 
The development of chronic headaches may be related to a progressive deterioration in the pain control system in patients with a daily intake of analgesics, which results in a potential habituation cycle (fig. 1). It has also been suggested that certain psychodynamic factors play a role in the development of analgesic abuse, with abusers having lower scores in psychological tests for anxiety and hysteria than non-abusers [3]. In the case of ergotamine, it has been suggested that a physical dependency may exist [5].

\section{Treatment of Migraine}

Non- Prophylactic medical

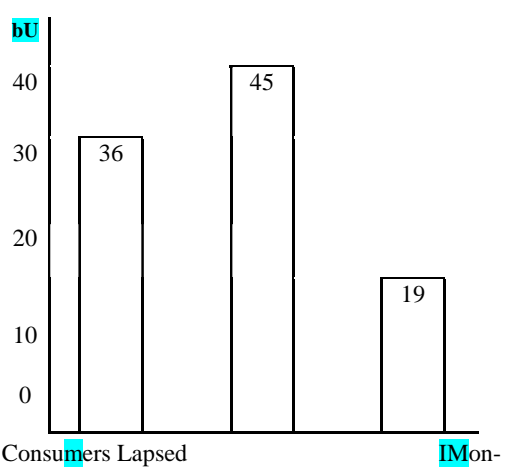

Although the majority of migraineurs were not consulting physicians about their headaches, they did use migraine therapy (fig. 2b). Nearly $50 \%$ were taking or had taken nonmedical therapies such as acupuncture or herbal remedies. Over 90\% had taken over-the-counter analgesics (which included codeine), while 8\% had taken prophylactic medication, and $44 \%$ had taken prescription medication. Of the prescription medications in current use, the majority (75\%) were analgesics (table 3). The predominance of analgesics among prescribed medicines is worrying, especially as a high percentage of patients must also be using over-the-counter analgesics. The high prevalence of use of analgesics among migraine patients may expose them to the risk of developing chronic daily headaches.

Fig. 1. Potential habituation cycle with over-use of simple analgesics. Adapted with permission from Findley et al. [13].

Fig. 2. (a) Consultation behaviour and (b) use of therapy by migraine sufferers in a Canadian study. Therapy includes both past and present use ( $\mathrm{n}=138)$. 


\section{Effective Treatment of Migraine}

Good treatment can start only with a confident, accurate diagnosis. It is imperative that the patient has confidence in the clinician and the diagnosis. The patient and

Edmeads

Migraine: a Clinician's View

Table 3. Use of prescribed medications by Canadian migraine sufferers

Medication

Percentage of patients (n=138)

Butalbital/acetylsalicylic/acid/caffeine Paracetamol/codeine

Other analgesics

Antidepressants

Ergotamine

Prophylactics

Other

tan for the acute treatment of migraine attacks may be more appropriate; long-term studies have shown that sumatriptan remains effective and, importantly, there are no indications that it has any intrinsic properties that encourage overuse [12].

The clinician's aim should be to establish, with the patient, a treatment regimen under which the headaches no longer significantly adversely affect quality of life. Once this has been done, there should be a continuous, but diminishing, follow-up with the ultimate aim of establishing the patient's independence from the physician.

Total $>100 \%$ because some patients were taking more than one type of prescription medication. Data from Edmeads et all. [9].

clinician can then work together to identify factors that trigger the headaches and then avoid or reduce them as far as possible. Non-pharmacological methods should be considered, for example stress reduction, or the appropriate use of local cold compresses or pressure. Prophylactic medication may be helpful for some patients, for example those with frequent attacks, or with menstrual migraine, or in whom acute therapies are medically contraindi-cated. When prescribing acute medication, the potential problem of chronic daily headaches in relation to analgesics and ergotamines should be considered and discussed with the patient. For many patients the use of sumatrip-

\section{Conclusions}

Migraine presents a major diagnostic and therapeutic challenge to general physicians and neurologists. Studies have shown that there is a general disenchantment of migraine patients with their physicians [8, 9] and a widespread problem of analgesic overuse [9].

These problems may be overcome by programmes of education for the public, physicians and neurologists. Patients should be made aware of the dangers of analgesic abuse and of the availability of appropriate and more effective migraine treatments. Neurologists should support their referral base - the physician - by providing information on procedures for the correct diagnosis of migraine, and on appropriate primary treatment. Finally, neurologists themselves must keep abreast of advances in the understanding of the pathogenesis of migraine and of advances in the treatment of this condition.

\section{References}

Headache Classification Committee of the International Headache Society: Classification and diagnostic criteria for headache disorders, cranial neuralgias and facial pain. Cephalalgia 1988;8(suppl7):19-28. Edmeads J: Headache and facial pain; in Stein JH (ed): Internal Medicine (4th edition). St. Louis, Mosby, 1994, pp 1025-1033. Micieli G: Analgesic abuse: the hidden problem in migraine; in: Migraine Through Expert Eyes. Worthing, PPS Europe Ltd., 1992, pp 49-52.

Mathew NT, Stubits E, Nigam MP: Transformation of episodic migraine into daily headache: analysis of factors. Headache 1982;22: 66-68.

5 Saper JR, Jones JM: Ergotamine tartrate de- 10pendency: features and possible mechanisms.

Clin Neuropharmacol 1986;9:244-256. 
6 Saper JR: Ergotamine dependency: a review. 11 Headache 1987;27:435-438.

Rapoport A, Weeks R, Sheftell F: Analgesic rebound headache: theoretical and practical implications. Cephalalgia 1985;5(suppl 3): 448-449. 12

8 Micieli G: Suffering in silence; in Edmeads J (ed): Migraine: A Brighter Future. Worthing, Cambridge Medical Publications, 1993, pp 1- 137.

Edmeads J, Findlay H, Tugwell P, Pryse-Phil-lips W, Nelson RF, Murray TJ: Impact of migraine and tension-type headache on lifestyle, consulting behaviour, and medication use. Can J Neurol Sci 1993;20:131-137.

Ekbom K, Ahlborg B, Scheie R: Prevalence of migraine and cluster headache in Swedish men of 18. Headache 1978;18:9-19. Rasmussen BK, Jensen R, Olesen J: Impact of headache on sickness, absence and utilization of medical services: Ekbom K, Ahlborg B, Scheie R: Prevalence of migraine and cluster headache in

Lloyd K: The clinical profile of sumatriptan: safety and tolerability. Eur Neurol 1994:34 (suppl 2):40-43.

Findley L, Henry P, Diener HC: Are traditional migraine therapies inadequate?; in Edmeads J (ed): Migraine: A Brighter Future. Worthing, Cambridge Medical Publications, 1993, pp 28-41. 